\title{
Hormiscia neu definiert
}

\author{
Peter Kornmann \\ Biologische Anstalt Helgoland, Meeresstation, Helgoland
}

\begin{abstract}
Hormiscia new-defined. There is only one way to clarify taxonomy and nomenclature of the Codiolum-Urospora-complex, and that is to compare cultures grown from the filamentous phase as well as from the unicellular stage. In this way correlative members in the life-history can be known, and the specifity or synonymy of the species is established. In the present study Codiolum gregarium A. Braun, described 1855 from Helgoland, is shown to be the sporophyte of Hormiscia neglecta, a species that was hitherto not distinguished from Hormiscia penicilliformis. The filaments of these two species grow consociated in the same localities; they are similar in shape but differ clearly in the size of their anisogametes and sporophytes. In addition, only the sporophyte of Hormiscia neglecta has been found in the field, which is quite remarkable as the sporophyte of both species grew vigorously in culture. The name Hormiscia is applied here to the anisogamous species which are filamentous when cultivated at $15^{\circ}$ or $5^{0} \mathrm{C}$. Urospora is restricted only to species without gametes; they are filamentous at low temperature and modify into dwarf-plants at $15^{\circ} \mathrm{C}$. The Codiolum-phase originates from asexual biflagellate zoospores of this stage.
\end{abstract}

\section{EINLEITUNG}

Der vorliegenden Studie liegt eine zufällige Beobachtung zugrunde: im November 1965 wurden zwei Codiolum-Formen von ganz verschiedenem Habitus nebeneinander am gleichen Standort gefunden. Das Kulturexperiment bestätigte die naheliegende Annahme, daß sie in den Entwicklungszyklus verschiedener Urospora-Arten gehörten. Damit war die Aufgabe gestellt, das Gebiet von Helgoland nach weiteren CodiolumFormen zu durchsuchen, den jahreszeitlichen Rhythmus ihres Auftretens und die Stadien ihrer Entwidklung festzustellen. Gleichzeitig wurde auch die fädige Urospora-Phase eingehend untersucht. Nach diesen umfangreichen Standortbeobachtungen und Kulturversuchen im Laboratorium können meine früher (KORNMANN 1961) mitgeteilten Ergebnisse über die Biologie von Codiolum und Urospora ganz wesentlich erweitert werden.

Fortpflanzung und Entwicklung der bisher untersuchten Urospora-Arten sind so verschiedenartig, daß mir die Aufgliederung dieser Gattung berechtigt erscheint. Urospora wormskioldii ist Vertreter einer Gruppe, in der eine geschlechtliche Differenzierung nicht bekannt ist. Die Erscheinungsform dieser Arten hängt von der Temperatur $\mathrm{ab}$ : ein Fadenstadium bei tiefer Temperatur modifiziert sich zu einem Zwergpflanzenstadium bei höherer Temperatur. Nur dieses bildet zweigeißelige ungeschlechtliche 
Schwärmer, aus denen die Codiolum-Phase entsteht. Für die Arten dieser Gruppe wird der Gattungsname Urospora beibehalten.

Die bisher als Urospora penicilliformis bezeichnete Art ist Typus einer Gruppe mit anisogamer Fortpflanzung. Das Codiolum-Stadium entsteht aus Zygoten. Die fädige Erscheinungsform dieser Arten wird durch die Temperatur nicht verändert. Für sie wird der alte Gattungsname Hormiscia FrIes gewählt. Die Gattung Hormiscia ist bei Helgoland mit drei Arten vertreten, über die in der vorliegenden Arbeit berichtet wird. Codiolum gregarium wurde als Sporophyt einer bisher noch nicht beschriebenen Hormiscia-Art erkannt. Es ist nicht identisch mit dem in den Lebenszyklus von Urospora wormskioldii gehörenden Pflänzchen, das ich früher für Codiolum gregarium hielt.

Isogamie ist bisher nur bei einer Form bekannt, für die ich die problematische Bezeichnung Urospora speciosa (CARm.) Leblond ex Hamel anwandte (KornmanN 1961). Auf diese Art wird in einer späteren Mitteilung noch zurückzukommen sein.

\section{ERGEBNISSE}

\section{Hormiscia penicilliformis (ROTH) FRIES}

Die Entwicklung dieser bei Helgoland häufigsten Form wurde bereits 1961 geklärt. Die damals angelegten Kulturen waren noch vorhanden und konnten in die vergleichenden Prüfungen der vorliegenden Untersuchung einbezogen werden. Ein sicheres Merkmal, das Hormiscia penicilliformis von einer häufig mit ihr vermischt vorkommenden Art unterscheidet, ist die Ausbildung der Fadenbasis kultivierter Pflanzen. Abbildung 1 zeigt die Unterschiede: Hormiscia penicilliformis bildet eine große Anzahl lang auswachsender Adventivrhizoiden aus. Das Haftorgan von Hormiscia neglecta dagegen ist klein und unscheinbar. Dieser Bestimmungsmethode haftet freilich der Nachteil an, daß aus einer eingesammelten Probe einzelne Fäden beziehungsweise Fragmente für Kulturen isoliert werden müssen. Im allgemeinen enthalten die Fäden bereits Zoosporangien, vegetative Fäden werden in Nährlösung bei $15^{\circ} \mathrm{C}$ meist schon nach wenigen Tagen fertil. Es ist zweckmäßig, nur wenige Zoosporen in die Kulturen einzubringen, damit sich die Fäden gut entwickeln können. Bei $15^{\circ} \mathrm{C}$ werden die Fäden im allgemeinen schon frühzeitig fertil, dagegen erhält man lange vegetative Fäden bei $5^{\circ} \mathrm{C}$. An 14 Tage alten Kulturen läßt sich die unterschiedliche Ausbildung der Fadenbasis bereits deutlich erkennen; bei einiger Erfahrung helfen auch weitere Merkmale, wie Farbe und Wuchsform der Fäden, die Versuchsdauer für eine sichere Unterscheidung der Arten zu verkürzen.

Fäden mit Gametangien wurden bei den häufigen Exkursionen im Jahre 1966 mehrfach gefunden; sie kommen also doch häufiger und auch während eines längeren Zeitraumes vor, als nach meinen früheren Angaben angenommen werden konnte. Sie waren im Februar und März 1961 in dem grünen Saum an der Eintauchlinie einer Boje gefunden worden. Von Ende Mai bis Anfang August 1966 wurden Geschlechtspflanzen wiederholt an Steinen an der Niedrigwasserlinie, Anfang Juli an Bojen und im Juli und August in einer breiten Zone unter der Hochwasserlinie gefunden. Hor- 
miscia penicilliformis ist diözisch; die männlichen Fäden sind leicht an der orangeolivgrünen Färbung ihrer Gametangien zu erkennen. Die weiblichen Gametangien sind nicht ohne weiteres von den Zoosporangien zu unterscheiden. Ein Faden enthält in der Regel zugleich Gametangien und Zoosporangien, wobei die einen oder die anderen in einzelnen Abschnitten überwiegen können.

Um Gameten zu erhalten, hat es sich bewährt, kleine Mengen des frisch gesammelten fertilen Materials in einem Tropfen Nährlösung auszubreiten und etwa 12 Stunden lang bis zur Beobachtung dunkel zu halten. Im allgemeinen schwärmt ein Teil der
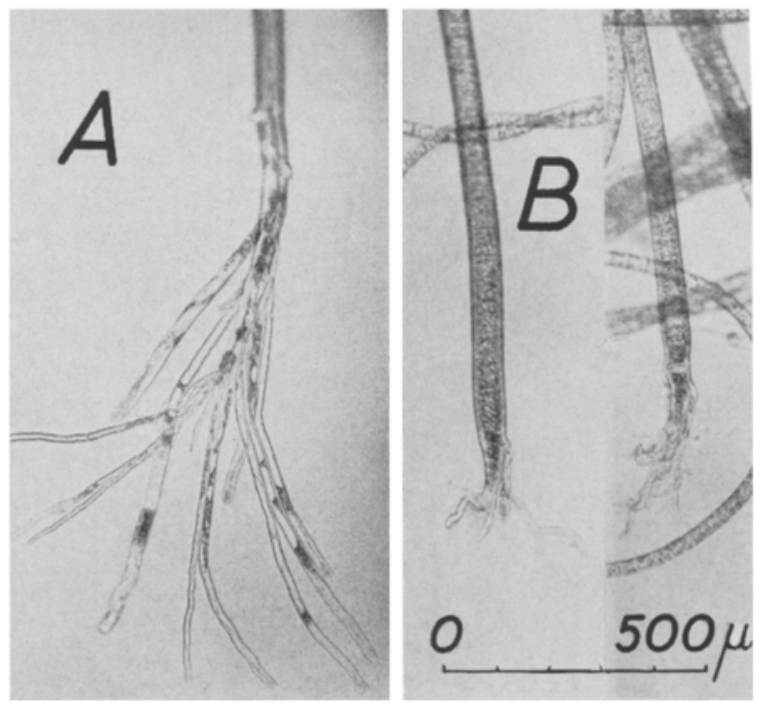

Abb. 1 : Basis 5 Wochen alter, bei $5^{\circ} \mathrm{C}$ kultivierter Fäden. A Hormiscia penicilliformis. $B$ Hormiscia neglecta nov, spec.

reifen männlichen Gametangien schon während dieser Zeit aus. Der Reiz des Lichtes und die Zugabe einiger Tropfen frischer Nährlösung regen die Entleerung der weiblichen und weiterer männlichen Gametangien an. Die Zoosporangien schwärmen erst später aus, so daß sich der Kopulationsvorgang leicht beobachten läßt und Zygoten erhalten werden können, denen keine oder nur wenige Zoosporen beigemischt sind. Es ist immer wieder eindrucksvoll, die Entleerung weiblicher Gameten in die bereits von vielen männlichen Gameten erfullte Flüssigkeit zu beobachten. Nach wenigen Sekunden sieht man nur noch anisogame Pärchen herumschwimmen (Abb. 2 A). Aus den Zygoten entwickeln sich rasch die kleinen kugeligen oder länglich-eiförmigen Codiolum-Sporophyten mit kurzem Stiel (Abb. 3). Einzelne können schon nach 8 Tagen fertil werden, die meisten schwärmen im Alter von etwa 15 Tagen aus. Ein Teil der Sporophyten entleerte sich nicht, wurde aber auch während weiterer 2 bis 3 Wochen. nicht wesentlich größer.

Zu aufschlußreichen Ergebnissen führten Beobachtungen an Gameten aus Einzelfäden. Frisch entleerte weibliche Gameten sind länglich-oval mit abgerundetem Hinter- 
ende. Langsam und stetig schwimmend suchen sie die hellste Stelle in einem Tropfen auf. Sie bleiben mehrere Stunden beweglich, verändern aber ihre Gestalt schon bald nach dem Freiwerden. Zunächst werden sie schlank und spindelförmig, ihr Hinterende läuft in eine mehr oder weniger lange Spitze aus (Abb. $2 \mathrm{~B}$ ). In diesem Zustand waren
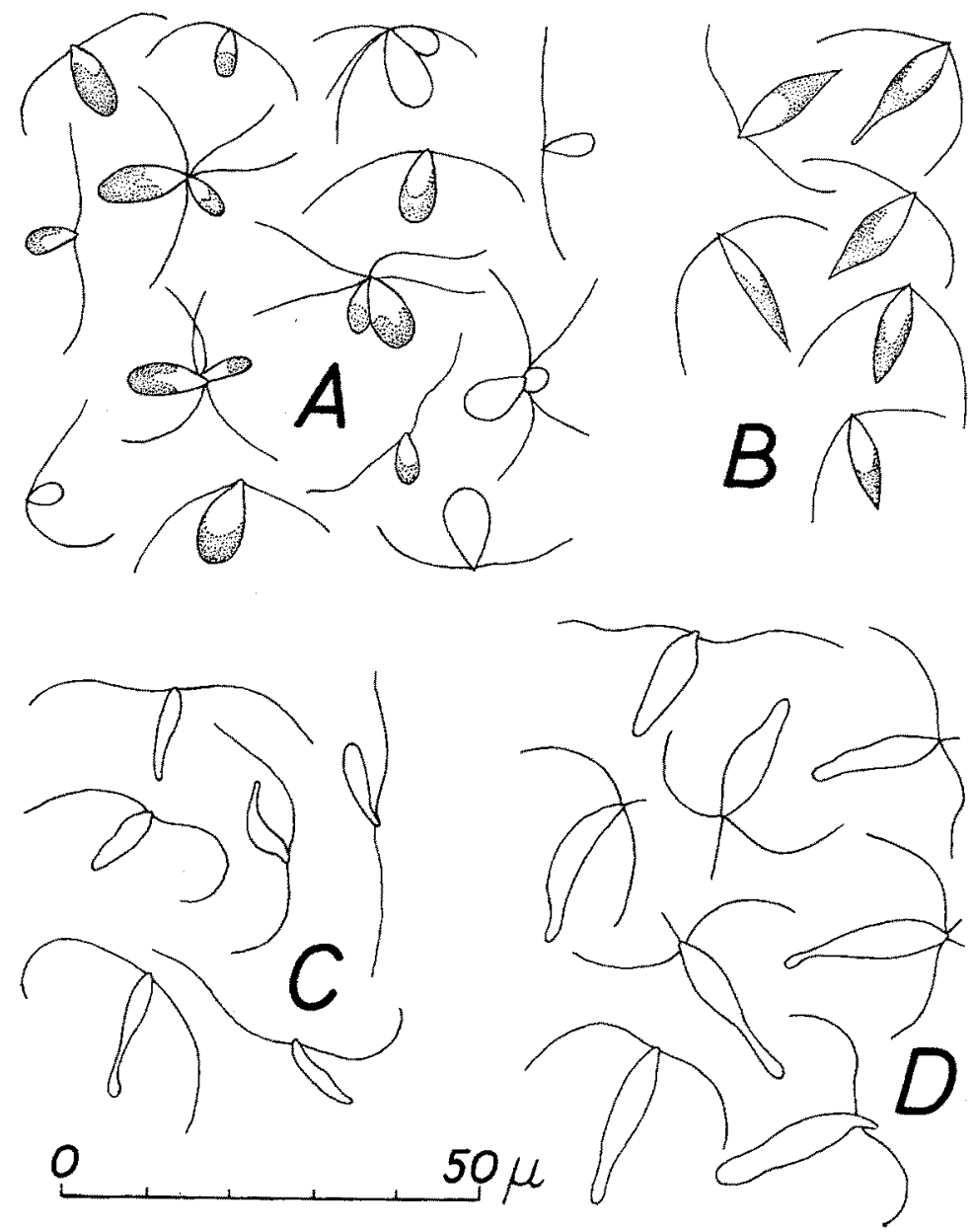

Abb. 2: Hormiscia penicilliformis. A Gameten und Kopulanten. $B$ Weibliche Gameten kurze Zeit nach dem Ausschwärmen. $C, D$ Etwa 8 Stunden alte männliche beziehungsweise weibliche Gameten

die Gameten nicht mehr kopulationsfähig. Einige Stunden später sind sie nicht mehr spitz, bei vielen schwillt das Hinterende nach einer leichten Einschnürung nochmals an. Das Vorderende trägt dann außer den Geißeln nicht selten noch einen oder auch zwei dünne Fortsätze (Abb. $2 \mathrm{D}$ ). Auch die männlichen Gameten zeigen entsprechende Gestaltsveränderungen (Abb. $2 \mathrm{C}$ ).

Angaben über die Morphologie sollten sich daher stets auf frisch entleerte Ga- 
meten oder Kopulationspartner beziehen. Wenn diese naheliegende und einleuchtende Voraussetzung nicht beachtet wird, kann es zu Interpretationen kommen, bei denen die Anomalität zur Norm erhoben wird. Hanic (1965) bildet auf Tafel $14 \mathrm{~A}$ Kopulationsstadien der von ihm als Urospora wormskioldii bezeichneten diözischen Art ab. Die Kopulanten sind oval und unterscheiden sich in Form und Größe kaum von denen in meiner Abbildung $2 \mathrm{~A}$. HANIC schreibt auf Seite 58: "Type I designated as $U$. wormskioldii conforms very closely in gametangium color and gamete size to

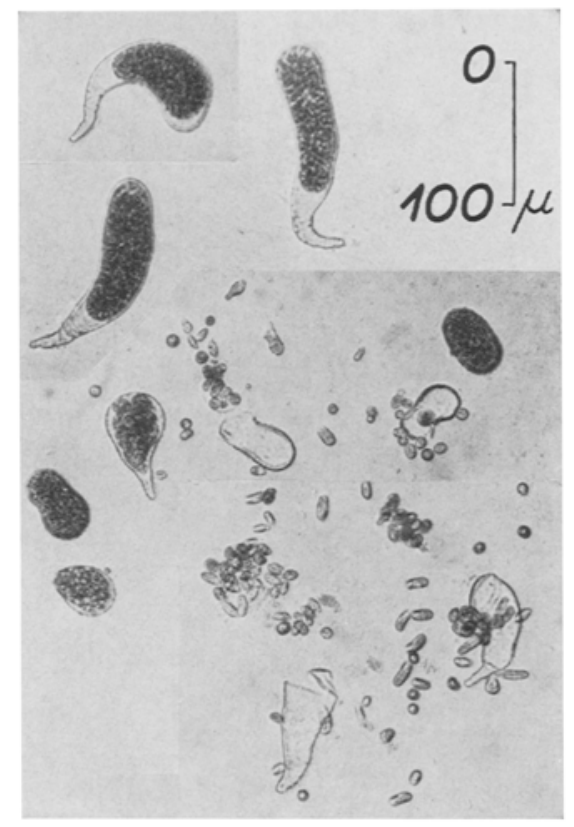

Abb. 3: Hormiscia penicilliformis. Reife und entleerte Sporophyten sowie Keimlinge. Aus einer 15 Tage alten Kultur bei $15^{\circ} \mathrm{C}$

U. penicilliformis from Helgoland (KORNMANN 1961c), but differs from it in that the male gametes are spindle shaped, the female gametes are asymmetrical and the male as well as the female has a pyrenoid (Fig. 22). In $U$. penicilliformis both gametes are ovale and only the female has a pyrenoid. However, these distinctions may not be valid. In $U$. wormskioldii the small pyrenoid of the male gamete is difficult to distinguish unless stained and the asymmetry of the female gamete is not assumed until some time after release. KoRNMANN did not stain his material nor did he observe gametes over long periods (personal communication). Future investigations of gamete morphology should take the time element into consideration." Diese Empfehlung sollte unbedingt beachtet werden, allerdings in dem Sinne, daß die Form der Gameten unmittelbar nach ihrem Austritt registriert wird, solange sie noch kopulationsfähig sind. Die Gestaltsänderung der nicht zur Kopulation gelangten Gameten ist zwar eine eigenartige Erscheinung, der aber keine besondere Bedeutung zukommt. 
Wahrscheinlich sind alle weiblichen Gameten zu parthenogenetischer Entwicklung fähig. Die so entstandenen Sporophyten unterschieden sich nicht von denen aus Zygoten, jedoch entstanden nur selten entwicklungsfähige Zoosporen. Auch im männlichen Geschlecht wurde "Parthenogenesis" beobachtet. Viele Tausende von Gameten sind in einem Tropfen enthalten, in dem ein männliches Fadenstiick ausschwärmt. Nachdem sie zur Ruhe gekommen sind, wird Nährlösung aufgefüllt. In allen Versuchen entwickelten sich im Bereich des ehemaligen Tropfens einige wenige Sporophyten. Wahrscheinlich entstehen sie aus einzelnen wesentlich größeren Schwärmern, die man zwischen den Gameten beobachten kann. Als parthenogenetische Entwicklung männlicher Gameten darf dieses minimale Auftreten von Sporophyten jedenfalls nicht angesehen werden.

Die Sporophyten von Hormiscia penicilliformis sind bisher nur aus Kulturen bekannt; in den vielen Codiolum-Proben, die im Laufe eines Jahres gesammelt und analysiert wurden, konnten sie niemals nachgewiesen werden. Auch dieser negative Befund unterscheidet Hormiscia penicilliformis von der mit ihr am gleichen Standort vorkommenden Hormiscia neglecta.

\section{Hormiscia neglecta nov. spec.}

Den ersten Hinweis auf diese häufig mit Hormiscia penicilliformis vermischt vorkommende Art gab die durch Abbildung 1 veranschaulichte unterschiedliche Ausbildung der Fadenbasis in den Nachkommenschaften von Einzelfäden aus ein und derselben Probe. Die Unterschiede sind sehr ausgeprägt: lange gerade, stelzenartige Rhizoiden bei Hormiscia penicilliformis gegenüber einer wenig auffälligen Verankerung bei der anderen Form. Die bei älteren Pflanzen aus den untersten Fadenzellen entspringenden Adventivrhizoiden schmiegen sich dem Faden an und breiten sich auf der Unterlage zu einem aus gewundenen Fädchen bestehenden Haftorgan aus.

Zur weiteren Kennzeichnung der neuen Art verhalfen Proben von Geschlechtspflanzen, die Anfang Juli 1966 im oberen Litoral gesammelt wurden. Sie enthielten zweierlei weibliche Gameten: auffallend große, die sich langsam und mit schraubigen Drehungen ungerichtet bewegten und die leicht von den wesentlich kleineren weiblichen Gameten von Hormiscia penicilliformis zu unterscheiden waren. Die großen Gameten kopulierten nicht mit den zahlreich vorhandenen schlank-ovalen männlichen Gameten, ihre Partner waren meist nahezu kugelig.

Aus diesen Proben wurde eine größere Anzahl von Fäden isoliert und zum Ausschwärmen gebracht. Die großen und die kleineren weiblichen Gameten entstammten verschiedenen Gametophyten. Nach Aufzucht der gleichzeitig entleerten Zoosporen ließen sich die Fäden mit den großen Gameten der in Abbildung $1 B$ dargestellten neuen Art zuordnen. Abbildung 4 zeigt Gameten und Kopulanten von Hormiscia neglecta. Die weiblichen Gameten variieren besonders in ihrer Dicke erheblich. Sie verändern ihre Gestalt in ähnlicher Weise wie die von Hormiscia penicilliformis. Nunmehr sind die Angaben von JoRde (1933) leicht zu verstehen: ganz offensichtlich waren auch in ihrem Untersuchungsmaterial die Gameten der beiden Arten vermischt. Nachdem sie erwähnt, daß Form und Größe der weiblichen Gameten sehr variabel 
sind, schreibt sie auf Seite 8: „Die kleinen Gameten werden in großer Anzahl in der Mutterzelle gebildet, die großen in kleiner Anzahl, zwischen 10 und 20. In zwei Nachbarzellen waren so in der einen Zelle 8, in der anderen 12 Gameten. Die Durchschnittsgröße der weiblichen Gameten beträgt $13 \mu$ Länge und $5,5 \mu$ Breite; die großen können aber $25 \mu$ lang und $9 \mu$ breit werden und sind dann im Volumen viel größer als

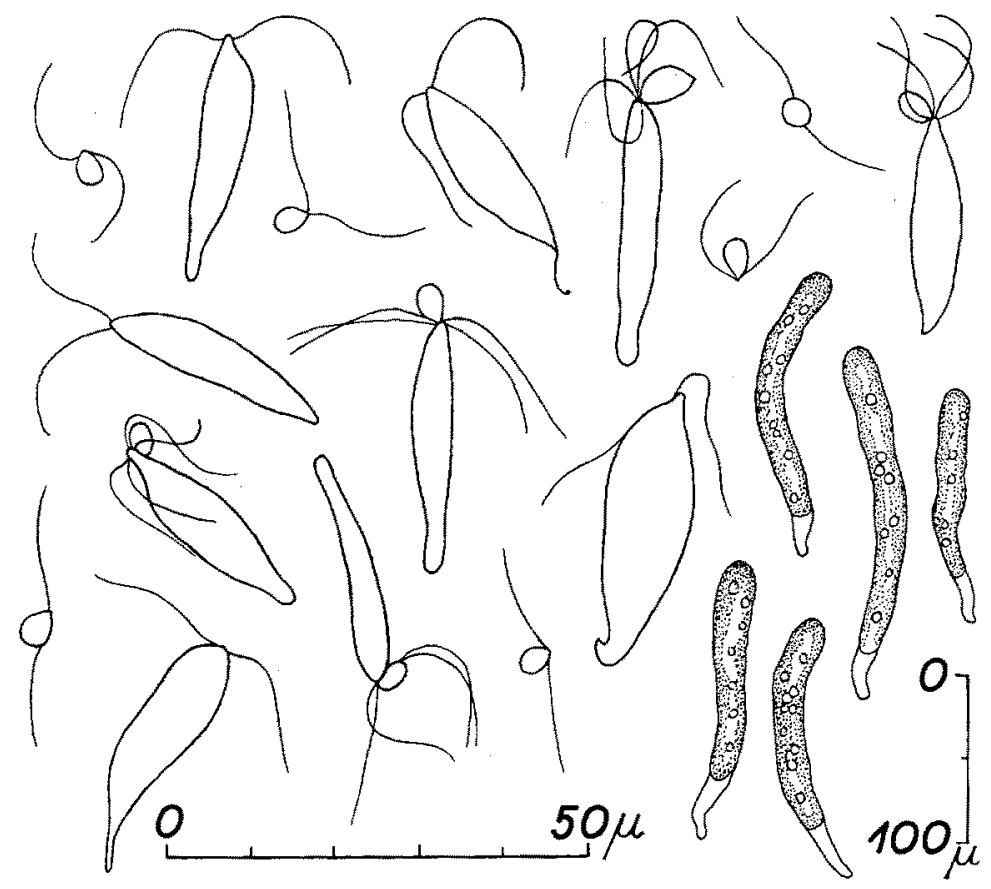

Abb. 4: Hormiscia neglecta nov. spec. Gameten und Kopulanten; in der unteren Ecke rechts Sporophyten im Alter von 14 Tagen

die Zoosporen. " Diese Angaben stimmen völlig mit meinen Beobachtungen überein, die größten weiblichen Gameten erreichten sogar $30 \mu$ Länge. Die männlichen Gameten sind kugelig bis oval und etwa 4,5 $\mu$ lang.

Schließlich zeigt auch die Sporophytenentwicklung von Hormiscia neglecta durchaus spezifische Merkmale. Bei keiner der anderen Hormiscia- und Urospora-Arten ließen sich in Kultur Codiolum-Pflanzen erzielen, die so wie diese in Größe und Habitus den am natürlichen Standort gewachsenen Sporophyten gleichkommen. Abbildung $5 \mathrm{~A}$ zeigt eine bei $15^{\circ} \mathrm{C}$ gewachsene vier Wochen alte Kultur. Sechs Tage später wurde der gleiche Bildausschnitt wieder photographiert (Abb. 5 B. Die Kulturflüssigkeit mußte abgegossen und ein Deckglas auf die ziemlich aufrecht wachsenden Pflänzchen gelegt werden. Es ließ sich daher nicht vermeiden, daß die Sporophyten sich etwas verschieden anordneten. Um den Vergleich der Bilder zu erleichtern, wurden Parallelen durch die Fußpunkte der beiden geraden Sporophyten eingezeichnet). Die Größenzunahme während der 6 Tage ist beträchtlich und beträgt bei dem langen geraden 
Pflänzchen etwa $300 \mu$. Am meisten hat sich der Stiel verlängert, obwohl er gar kein selbständiges Wachstum hat. Seine Verlängerung ist nur die Differenz zwischen dem Gesamtwachstum des Pflänzchens und dem des grüngefärbten Köpfchens. Das Codiolum-Pflänzchen wächst apikal, und der farblose Stiel wird an der Basis des Protoplasten abgeschieden. Durch diesen Wachstumsmechanismus erklärt sich auch der lamel-

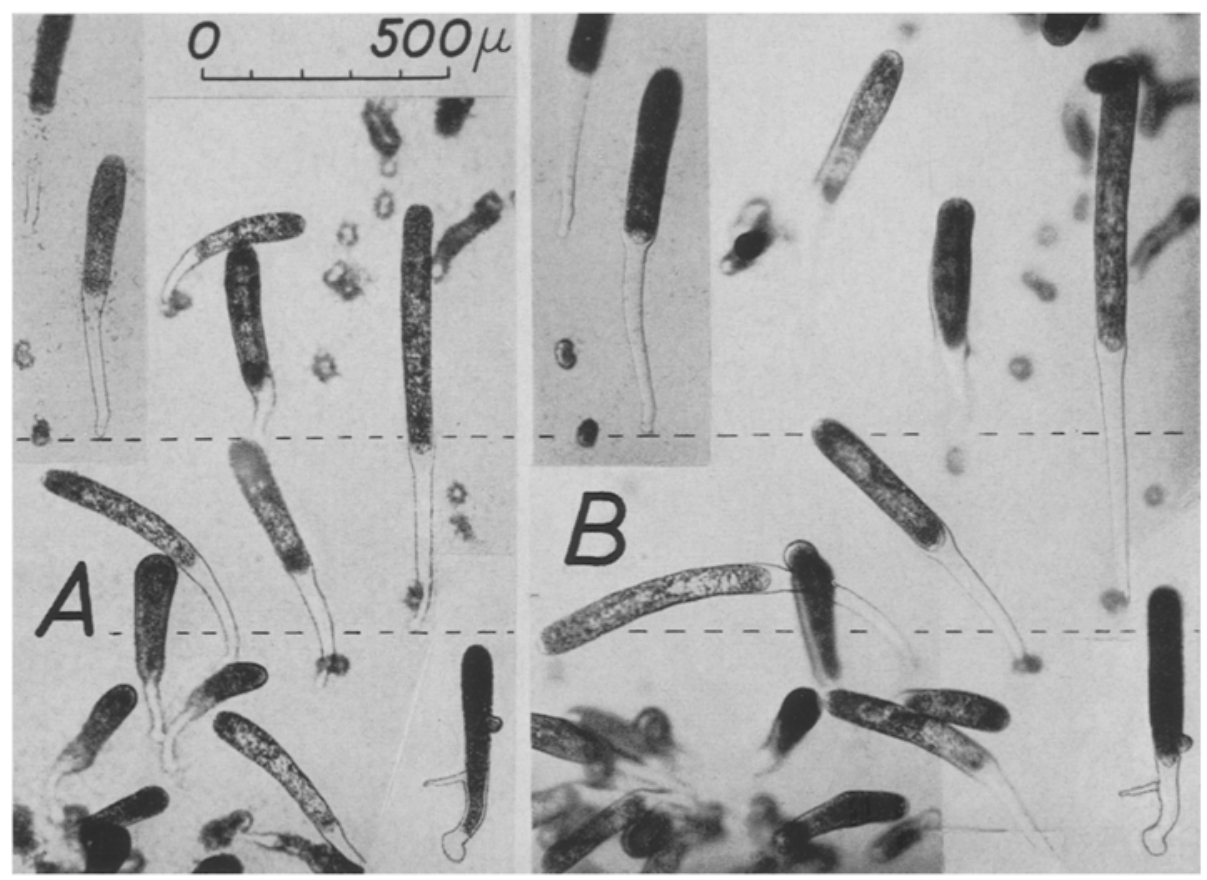

Abb. 5: Hormiscia neglecta nov. spec. Bei $15^{\circ} \mathrm{C}$ kultivierte Sporophyten. A 4 Wochen alt. $B$ Der gleiche Ausschnitt 6 Tage später

lare Aufbau des Stieles von Codiolum: seine Schichtung ist das Ergebnis rhythmischer, vermutlich tagesperiodischer Membranausscheidungen des Protoplasten. In gleicher Weise dürfte auch die Gliederung in den Stielchen der Sporophyten von Spongomorpha aeruginosa zustande kommen. $\mathrm{Da}$ diese Pflänzchen in Kultur sehr schnell wachsen, konnte der Eindruck einer Septierung der Stielchen entstehen (KorNMANN 1964).

Das apikale Wachstum von Codiolum wird sehr anschaulich an dem jeweils in der rechten unteren Ecke eingefügten Pflänzchen gezeigt. Es ist durch einen dornartigen Membranfortsatz "markiert"; solche Fortsätze kommen wie bei Codiolum petrocelidis mitunter auch an den Sporophyten von Hormiscia neglecta in Kultur vor. Während der sechs Tage hat sich der grüne Inhalt bis oberhalb der Ansatzstelle des Dorns verlagert. Die Sporophyten in Abbildung $5 B$ verlängerten sich in den folgenden Tagen nur noch wenig, doch wurden die Köpfchen dicker und zum großen Teil auch fertil.

Hormiscia neglecta ist diözisch, die Gameten beider Geschlechter können sich in 


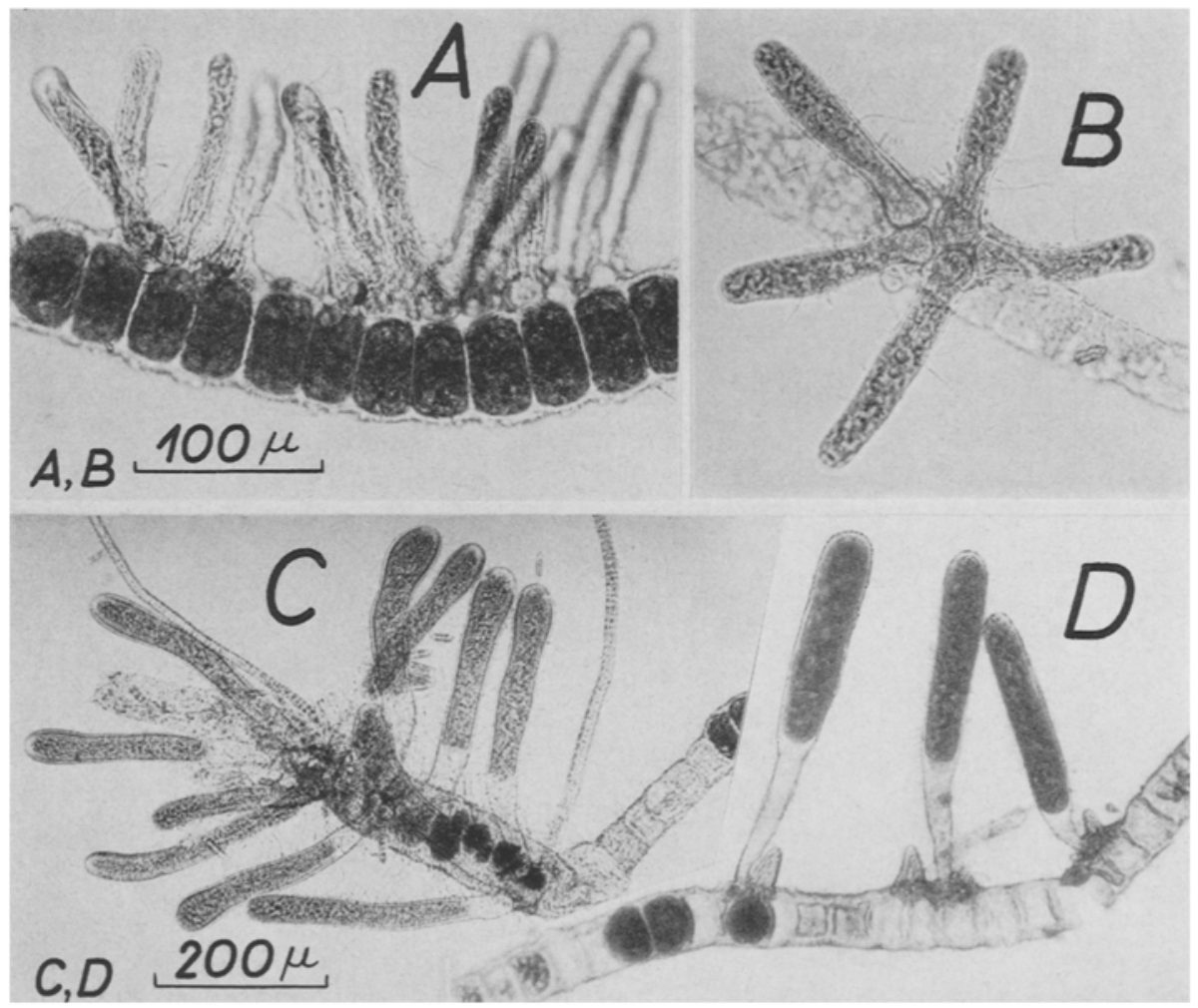

Abb. 6: Codiolum gregarium, epiphytisch auf Fäden von Hormiscia. A, $B$ Noch nicht in Stiel und Kopf differenzierte Sporophyten (14. Juni 1966). C Gestielte Pflänzchen (9. Juni 1966). $D$ Die größten epiphytischen Codioli (7. Juli 1966). Der Wirtsfaden trägt auch einige junge Blidingia-Pflänzchen

der gleichen Weise parthenogenetisch entwickeln, wie es bereits bei Horrniscia penicilliformis geschildert wurde. Mit unverschmolzenen Gameten der beiden Arten angelegte Kulturen lassen sich leicht an den charakteristischen Unterschieden ihrer parthenogenetisch entstandenen Sporophyten erkennen.

\section{DIAGNOSE}

\section{Hormiscia FrIES emend.}

Genus Codiolacearum. Filamentis simplicibus e cellulis multinucleatis constitutis. Propagatur asexualis per formationem zoosporarum in inferiore parte cuspidatarum. Cellulis sexualibus dimorphis.

Gattung der Codiolaceen. Unverzweigte Fäden mit vielkernigen Zellen. Ungeschlechtliche Vermehrung durch Zoosporen mit spitzem Hinterende. Geschlechtszellen anisogam. 
Hormiscia neglecta nov. spec.

Gametophytum dioicum, cuius sporophyta A. BRAUN (1855) ad insulam Helgoland collegit et Codiolum gregarium nominavit. Habitat eodem loco consors speciei Hormiscia penicilliformis, a qua filorum viriditate paulo clariore exigue differt. Filamenta sexualia colligebantur in summa parte litoris maritimi mensibus Julio et Augusto. Macrogametis ellipsoideo-elongatis, (20) - $25-(30) \mu$ longis, microgametis ovato-rotundis, $4,5 \mu$ longis.

Diözischer Gametophyt, dessen Sporophyten A. Braun (1855) bei Helgoland sammelte und als Codiolum gregarium beschrieb. Wächst am gleichen Orte zusammen mit Hormiscia penicilliformis, von der sie sich durch die etwas mehr hellgrüne Farbe ihrer Fäden nur wenig unterscheidet. Geschlechtspflanzen wurden im Juli und August im obersten Teil der Litoralzone gesammelt. Makrogameten langgestreckt, (20) - 25 (30) $\mu$ lang, Mikrogameten rundlich-oval, 4,5 $\mu$ lang.

\section{Codiolum gregarium A. BRAUN}

Nach den eingehenden Beobachtungen über die im Gebiet um Helgoland vorkommenden Codiolum-Formen kann kein Zweifel daran bestehen, daß das von BRAUN (1855) beschriebene Codiolum gregarium mit dem Sporophyten von Hormiscia neglecta identisch ist. Für diesen Nachweis bedarf es nicht einmal der Prüfung des Originalmaterials. Braun hat über seine im Herbst 1852 bei Helgoland gefundene Form bereits am 22. September des gleichen Jahres in einem Vortrag berichtet. Zu dieser Jahreszeit ist aber das zum Formenkreis von Urospora wormskioldii gehörende Codiolum noch so spärlich und klein, daß es überhaupt nicht auffällt. Die im August und September bei Helgoland vorkommenden samtartigen Codiolum-Rasen sind ausschließlich die Sporophyten von Hormiscia neglecta. Es erschien mir zweckmäßig, dieses Ergebnis den folgenden Beobachtungen voranzustellen.

Die ersten jungen Codiolum-Pflanzen wurden am 9. Juni 1966 epiphytisch auf Fäden von Hormiscia gefunden, die auf einem Steinwall in der obersten Gezeitenzone der Düne wuchsen, einer flachen Insel $1,5 \mathrm{~km}$ östlich von Helgoland. Es waren grüne Schläuche, die mit etwas angeschwollener Basis auf den Wirtspflanzen hafteten (Abb. 6 $A, B)$. Der farblose Stiel bildet sich erst aus, wenn der grüne Inhalt den apikal wachsenden Schlauch nicht mehr ganz ausfüllt (Abb. $6 \mathrm{C}$ ). Bis zum 7. Juli konnten wiederholt Proben dieses epiphytischen Codiolums gesammelt werden, dann verschwanden die inzwischen überständig gewordenen Wirtspflanzen. Es waren immer schlauchartige Stadien neben gestielten Codiolum-Pflänzchen $\mathrm{zu}$ finden, was auf eine laufende Entstehung neuer Sporophytengenerationen schließen läßt. Eine Anzahl besonders großer Sporophyten aus einer Probe vom 7. Juli (Abb. $6 \mathrm{D}$ ) wurde isoliert und bei $5^{\circ} \mathrm{C}$ weiterkultiviert. Sie wurden unter diesen Bedingungen nach 3 bis 6 Wochen fertil; ihre Zugehörigkeit zu Hormiscia neglecta wurde durch das Kulturexperiment erwiesen. Sicherlich wären diese Sporophyten ebenso wie die aus Zygoten kultivierten auch bei $15^{\circ} \mathrm{C}$ reif geworden, jedoch wurden die Rohkulturen bei dieser Temperatur völlig von Cyanophyceen und Diatomeen uiberwuchert. 


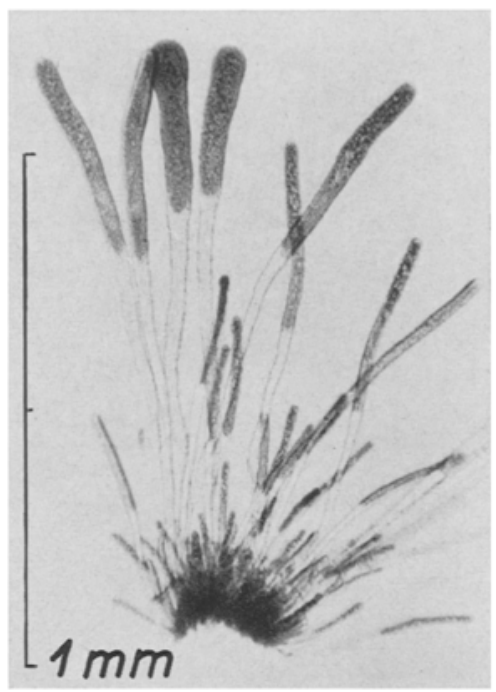

Abb. 7: Codiolum gregarium, Helgoland (26. Juli 1966). Die Probe enthält alle Stadien der Entwicklung: undifferenzierte Schläuche, dünne fadenförmige und leicht keulig verdickte Sporophyten

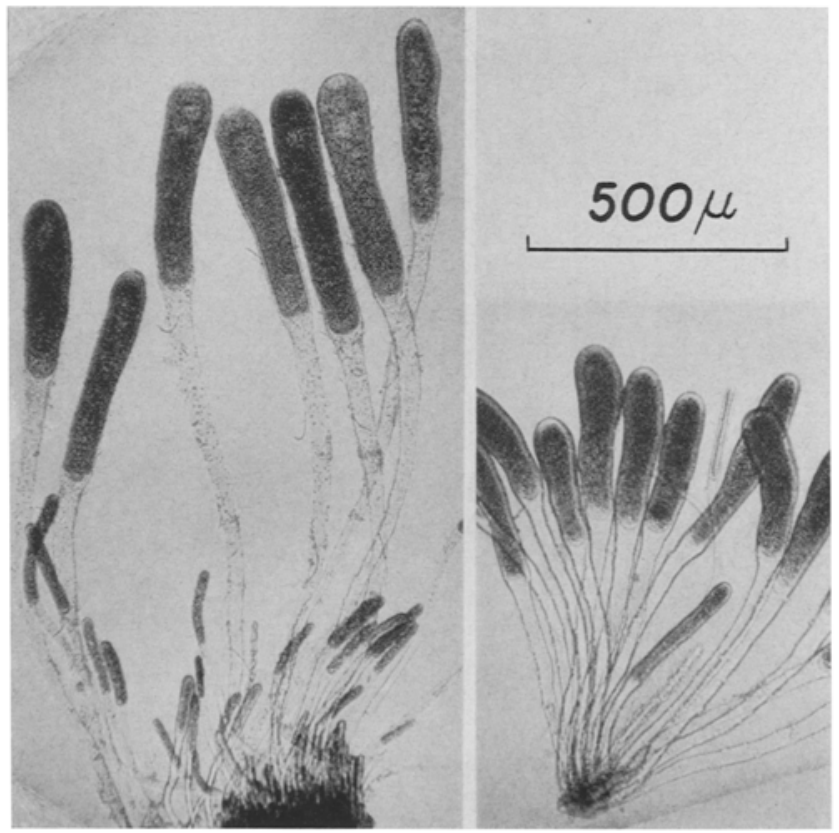

Abb. 8: Codiolum gregarium, Helgoland (24. August 1966). Zwei Proben von unterschiedlichem Habitus von benachbarten Steinen 
Kurze Zeit später, am 26. Juli, gaben sich samtige Uberzüge auf Granitsteinen des oben bezeichneten Fundortes als dichte Codiolum-Rasen zu erkennen (Abb. 7). Alle Entwicklungsstadien von den noch undifferenzierten grünen Schläuchen über die fadenförmigen, mehr oder weniger langgestielten Pflänzchen bis zu etwa $1 \mathrm{~mm}$ hohen Exemplaren mit leicht verdickten Köpfchen waren in diesen Proben zu finden. Vier Wochen später wurden die in Abbildung 8 dargestellten Proben gesammelt. Sie stammen von unmittelbar benachbarten Steinen, weichen aber in der Länge ihres Stieles

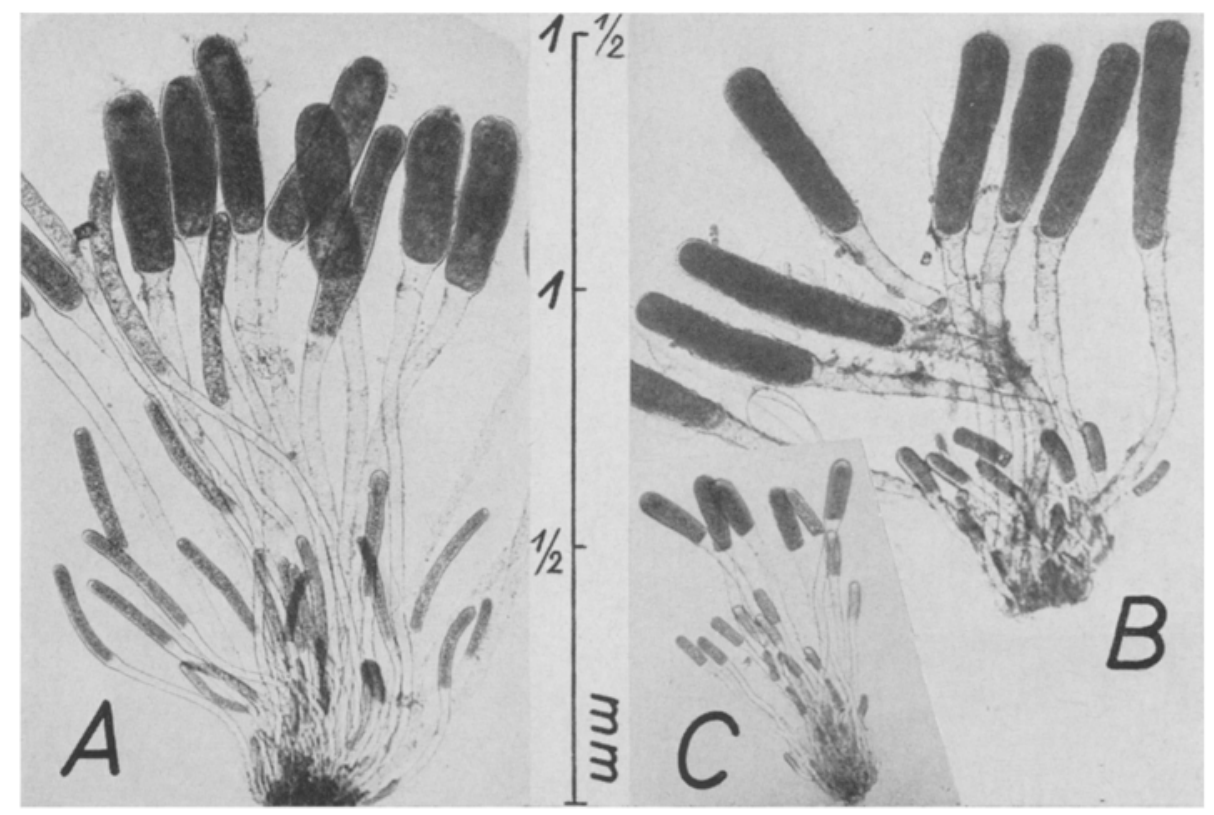

Abb. 9: A Codiolum gregarium, langstielige Pflanzen in allen Entwicklungsstadien. B Codiolum gregarium, kürzer gestielt. Die jungen Pflänzchen am Grunde des Büschels gehören wie die bei $C$ za einer Form von Urospora wormskioldit. Sie sind in allen Stadien ihrer Entwicklung deutlich von Codiolum gregarium verschieden. Alle Proben von Helgoland (8. September 1966)

sehr voneinander ab. Die besonderen Verhältnisse des jeweiligen Substrates, seine Exposition, Neigung, Beschattung und Niveauhöhe, dürften die Variation der Pflänzchen bewirken. Auf der Suche nach dem Sporophyten von Hormiscia penicilliformis wurde auf solche morphologisch extremen Codiolum-Pflanzen besonders geachtet. Die Prüfung im Kulturversuch ergab jedoch immer nur die Zugehörigkeit zu Hormiscia neglecta.

Proben vom 8. September (Abb. $9 A, B$ ) passen genau zu der Beschreibung, die Braun $(1855$, p. 21-22) seinem Codiolum gregarium gab: „Specimina adulta et perfecta, $1-1^{1 / 2} \mathrm{~mm}$ longa, clavam habent oblongam $1 / 4-1 / 2$, plerumque $1 / 3 \mathrm{~mm}$ longam, $1 / 15-1 / 11 \mathrm{~mm}$ crassam et stipitem clava $1^{1 / 2-3}$ longiorem, superne $1 / 20-1 / 18 \mathrm{~mm}$ crassum, deorsum sensim attenuatum, versus basin $1 / 45-1 / 35$ crassum, ..." Diesen Maßen entsprechen genau die großen Sporophyten in den Abbildungen $9 A$ und $B$. Mit voller 
Absicht wurde für die Abbildung $9 B$ eine Probe gewählt, die neben Codiolum gregarium die jungen Stadien eines andersartigen Codiolums enthält. Diese Form wurde am 8. September zum ersten Male sowohl zwischen Codiolum gregarium wachsend als auch reine Räschen bildend gefunden (Abb. $9 \mathrm{C}$ ). Schon die jüngsten Stadien unterscheiden sich durch die sehr viel kürzeren Köpfchen auf einem glasklaren Stiel deutlich

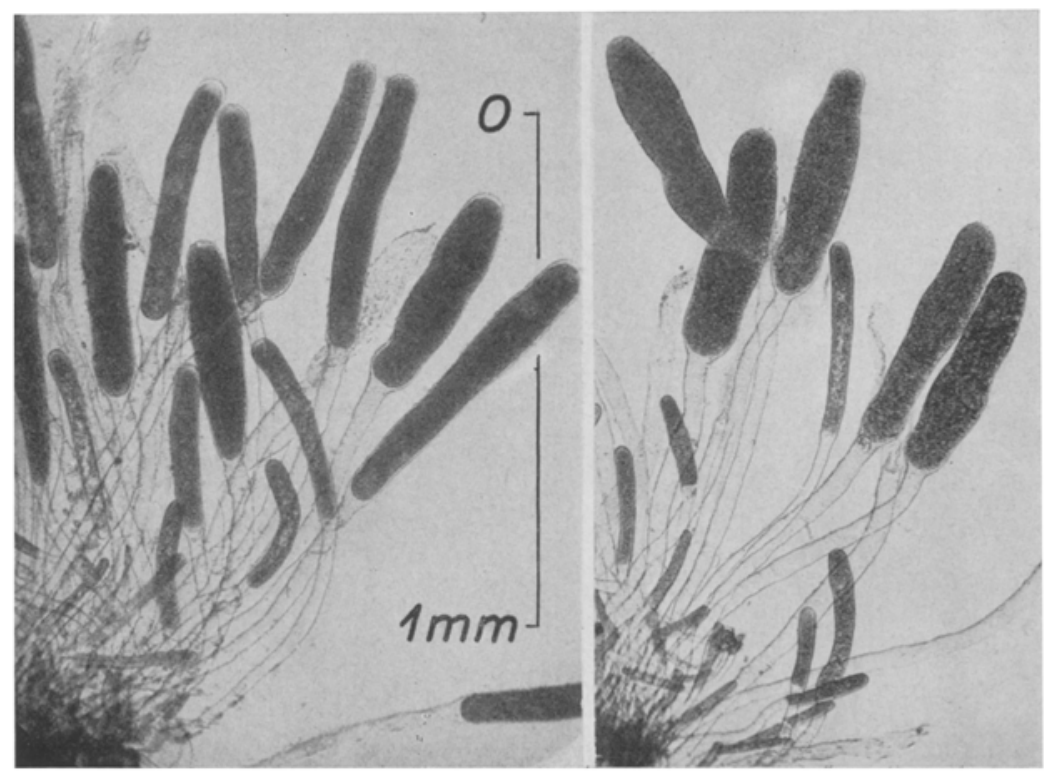

Abb. 10: Codiolum gregarium, Helgoland (28. 10. 1966). Vegetative, reife und entleerte Sporophyten

von vergleichbaren Stadien der neglecta-Sporophyten (Abb. 9 A). Bei $5^{0} \mathrm{C}$ kultiviert, wurden isolierte Pflänzchen Anfang Oktober fertil. Sie gehören in den Formenkreis von Urospora wormskioldii.

Ein großer Teil der Sporophyten von Hormiscia neglecta wird im Oktober fertil. Die beiden Ausschnitte in Abbildung 10 zeigen die Variabilität der reifen Pflanze anschaulicher, als alle Beschreibungen und Maßangaben sie vermitteln könnten. Zum Vergleich sind in Abbildung 11 die inzwischen herangewachsenen Codioli von Urospora wormskioldii dargestellt; der unterschiedliche Habitus schließt jede Verwechslung aus.

In der zweiten Oktoberhälfte entleert die Mehrzahl der Sporophyten von Hormiscia neglecta ihre Schwärmer, und im November verschwindet das Pflänzchen immer mehr aus dem Vegetationsbild. Die gleichen Steine, die früher von Codiolum gregarium besiedelt waren, tragen dann einen dunkelgrünen samtartigen Uberzug, das wormskioldii-Codiolum. Einzelne geringfügige Reste der neglecta-Sporophyten geben sich schon dem unbewaffneten Auge als bräunlich-grüne Überzüge zu erkennen. Diese Färbung wird durch eine starke Besiedelung der leeren Sporangienhüllen durch Diatomeen und Cyanophyceen verursacht. In solchen Rasen findet man noch vereinzelte 
nachgereifte neglecta-Sporophyten, deren Form ganz charakteristisch von den früher reifgewordenen abweicht (Abb. 12). Die schlankeren Keulen sind weniger scharf von den Stielen abgesetzt. Vermutlich ist diese Erscheinungsform der Sporophyten durch die jahreszeitlich veränderten Temperatur- und Lichtverhältnisse bedingt.

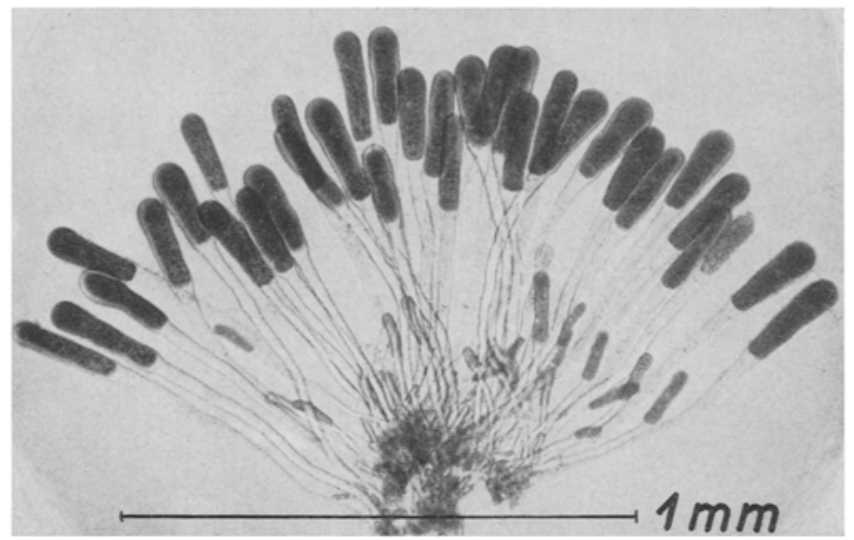

Abb. 11: Dieses Codiolum gehört zu einer Form von Urospora wormskioldii. Zum Vergleich mit Abbildung 10. Helgoland (28. Oktober 1966)

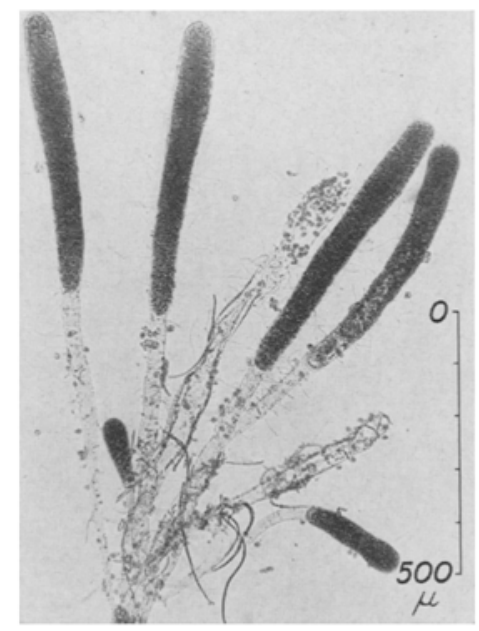

Abb. 12: Codiolum gregarium, Helgoland (19. November 1965). Nachgereifte, im Habitus vom Typus abweichende Pflanzen. Die beiden untersten Pflänzchen gehören zu Urospora wormskioldii

Die zahlreichen Photos lebenden Materials von Codiolum gregarium in allen Entwicklungsstadien kennzeichnen dieses so of in der Literatur genannte und verkannte Pflänzchen jetzt eindeutig. Nicht abgebildet sind die extrem langen Formen, bei denen allein die Stiele bis zu 1,6 mm Länge erreichen können. Die Zeichnungen 
von BRAUN (1855) lassen allerdings wenig Ahnlichkeit mit dem Objekt erkennen; sie sind sogar irreführend und entsprechen nicht seiner Beschreibung (vergleiche oben). Darauf wies schon Börgesen (1902) nach einer Prüfung von Herbarmaterial der Helgoländer Pflanze hin. Er stellte fest, daß zumindest bei vollentwickelten Exemplaren Stiel und Kopf nicht unmittelbar ineinander übergehen, sondern deutlich durch eine Einschnürung getrennt sind. Außerdem war der Stiel im allgemeinen länger als in Brauns Abbildung.

Codiolum gregarium wurde an mehreren Stellen von Helgoland gefunden. In rasenartigen Uberzügen besiedelt das Pflänzchen Urgesteinsblöcke eines Walles an der Ostküste der Düne dicht unter der Hochwasserlinie. Die samtartige Oberfläche der feuchten Rasen ist ebenso charakteristisch wic das eigenartige Bild der angetrockneten Bestände. Die Flächen werden beim Austrocknen in kleine unregelmäßige Felder in der Art von Trockenrissen aufgeteilt. Auch Collins (1909) machte auf diese Erscheinung aufmerksam, "characteristic and readily recognized, but not easy to describe".

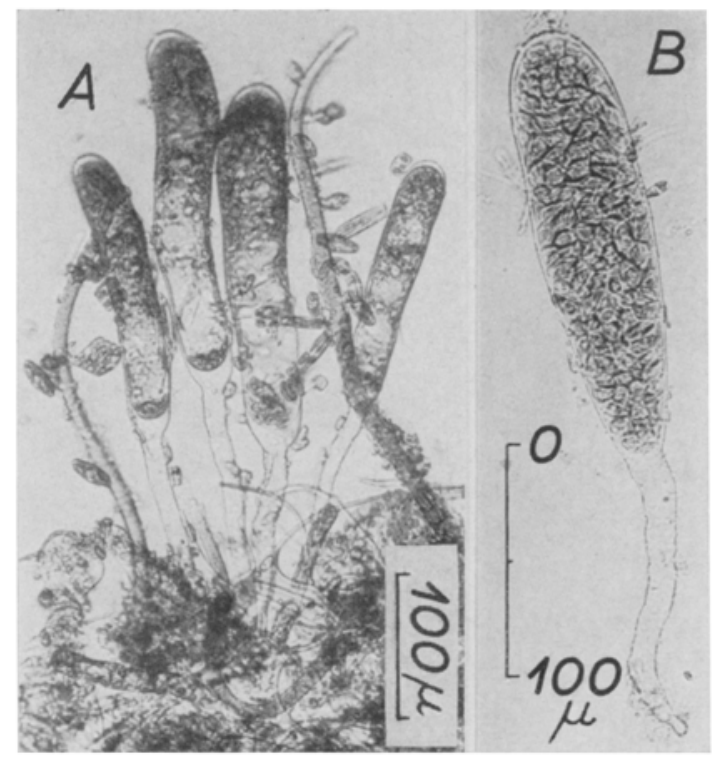

Abb. 13: Hormiscia spec., Helgoland (15. Dezember 1965). A Vegetative Gruppe.
\[ \text { Fertiler Sporophyt } \]

Hormiscia spec.

In den zahlreichen für diese Untersuchung gesammelten Proben konnte nur einmal der Sporophyt einer weiteren Hormiscia-Art gefunden werden. In einer Aufsammlung vom 15. Dezember 1965 waren einzelne kleine, zartwandige CodiolumPflänzchen in einen dichten Belag von Cyanophyceen, Diatomeen und fädigen Grünalgen auf Betonblöcken nahe der Hochwasserlinie eingestreut (Abb. 13). Die meisten 
Zellen waren vegetativ und zeichneten sich durch einen hellgrünen, locker netzigen Chromatophor aus. Einzelne Pflänzchen waren fertil (Abb. $13 B$ ) und entließen am 16. Dezember Zoosporen. Auch die von den Verunreinigungen weitgehend isolierten vegetativen Zellen wurden bei $5^{\circ} \mathrm{C}$ nach 3 bis 4 Wochen reif.

Die aus den Zoosporen kultivierte Fadengeneration ähnelt Hormiscia neglecta, ist aber viel mehr von den Temperaturbedingungen abhängig als diese Art. Sie wächst bei $5^{\circ} \mathrm{C}$ noch üppiger als Hormiscia neglecta; ihre langen Fäden winden sich zu dicken seilartigen Strängen zusammen. Bei $15^{\circ} \mathrm{C}$ dagegen bleiben sie kurz und werden frühzeitig fertil. Hier ist Hormiscia neglecta stark überlegen; zwar können die Endzellen ihrer jungen Fäden bei $15^{\circ} \mathrm{C}$ ebenfalls Zoosporen bilden, doch wächst die vegetative Basis dann noch beträchtlich in die Länge.

Das ausgeprägte Wachstumsoptimum bei tiefer Temperatur läßt darauf schließen, daß diese Art in kälteren Gewässern beheimatet ist und ihre Fäden sowie Sporophyten bei Helgoland sehr selten sind. Es wäre schon ein großer Zufall, die Fäden, die äußerlich keine rechten Unterscheidungsmerkmale gegenüber anderen Arten erkennen lassen, am Standort zu finden. Die Eingliederung bei Hormiscia erfolgt hier mit dem Vorbehalt, daß die Anisogamie noch nachgewiesen wird. Bisher sind noch keine Gametangien beobachtet worden, jedoch traten in einer Kultur bei $5^{\circ} \mathrm{C}$ einzelne CodiolumSporophyten auf, die vermutlich aus weiblichen Gameten entstanden sind. Auch in den Kulturen von Hormiscia penicilliformis traten gelegentlich mehr oder weniger viele Sporophyten auf. Das gleiche beobachtete HaNIC (1965) bei einem weiblichen Klon der von ihm als Urospora wormskioldii bezeichneten Art.

\section{SCHLUSSBETRACHTUNG}

Die vorliegende Studie zeigt den Weg, auf dem die Taxonomie der hauptsächlich in den kälteren Meeren verbreiteten Gattung Urospora im weiteren Sinne geklärt werden kann und - so darf man hoffen - auch geklärt werden wird. Die für Helgoland gewonnenen Ergebnisse lassen sich nicht ohne weiteres auf die Vegetationsverhältnisse anderer Küstenabschnitte übertragen; es bedarf jeweils des Kulturexperimentes, um Ubereinstimmungen oder Unterschiede mit den an anderen Orten gesammelten Formen festzustellen. Nur so lassen sich die Arten definieren und ihre Verbreitungsgebiete erfassen. Die bisher vorliegenden Beschreibungen nach morphologischen Merkmalen und die darauf gegründete systematische Gliederung der Gattung samt ihrer Nomenklatur ist nur von sehr beschränktem Wert. Das gilt für die beiden heteromorphen Partner dieser Arten, den fädigen Typus wie auch das Codiolum-Stadium. Es ist eine ganze Reihe von Codiolum- „Arten“ beschrieben worden. Einige Autoren (PRINTz 1926, HaNic 1965) hielten sie wie auch ich (KornmanN 1961) für Modifikationen von Codiolum gregarium. Diese Annahme hielt der exakten Nachprïfung nicht stand: über Spezifität oder Synonymie der Codiolwm-Formen kann nur das Kulturexperiment entscheiden.

HaNIC (1965) bildet auf Tafel 24 zwei Codiolum-Formen von sehr unterschiedlichem Habitus von den Küsten Britisch-Kolumbiens ab. Die von ihm als Codiolum gregarium bezeichnete Form sieht völlig anders aus als der Typus von Helgoland. 
Dick angeschwollene ovale Keulen sitzen den Stielen ganz unvermittelt auf. Wenig Abnlichkeit mit dem von den Färöer beschriebenen Typus LyngBYes (Abbildung bei Börgesen 1902) hat auch seine für Codiolum pusillum angesehene Pflanze. Die Beziehungen dieser Codiolum-Formen zu den in dem Untersuchungsgebiet vorkommenden und von HANIC ausführlich behandelten Urospora-Arten sind nicht geklärt worden. Sie hätten weitere Daten für einen Vergleich von HANICs Urospora wormskioldii mit Urospora penicilliformis erbringen können. Die beiden Arten sind einander sehr ähnlich, was bereits HaNic feststellte und ich nach Prüfung seines Originalmaterials bestätigen kann. Dagegen kann ich HANICs Angabe (p. 73) nicht bestätigen, daß sich die kultivierten Fäden von Urospora vancouveriana nicht von denen seiner Urospara wormskioldii unterscheiden sollen. In meinen Kulturen sind diese beiden Arten von ganz verschiedenem Habitus, außerdem unterscheiden sie sich klar von den Helgoländer wormskioldii-Formen.

In der vorliegenden Arbeit blieb die Morphologie der am natürlichen Standort gewachsenen fädigen Pflanzen bisher unberücksichtigt. Die Dicke der Fäden und Form ihrer Zellen variieren zu sehr, um als Bestimmungsmerkmal dienen zu können. Die auf solchen Angaben geschaffene Gliederung der Gattung ist völlig subjektiv und unbefriedigend. Von dieser Kritik kann auch meine 1961 erschienene Arbeit nicht ausgeschlossen werden. Ich hatte damals Urospora bangioides für Helgoland aufgeführt. Diese Art sollte durch ihre dickeren Fäden von Urospora penicilliformis unterschieden sein; ihre Vermehrung sollte nur durch Zoosporen erfolgen. Zu meiner großen Ubberraschung wurde die vermeintliche Urospora bangioides im April 1965 und im Mai 1966 auf Steinen an der Niedrigwasserlinie mit Gametangien gefunden. Das Kulturexperiment ergab die völlige Ubereinstimmung mit Proben, die dünnfädiger waren und als Hormiscia penicilliformis angesehen wurden. Urospora bangioides stellt offensichtlich eine Modifikation dar, bei der die Fäden unter den Bedingungen des Standorts und der niedrigen Wassertemperatur eine Breite bis zu $250 \mu$ erreichen können. Kulturen des 1961 untersuchten Materials von Urospora bangioides sind nicht mehr vorhanden. Die Fadenbasis sollte nämlich durch das Fehlen von Adventivrhizoiden von Hormiscia penicilliformis unterschieden sein. Diese Angabe läßt vermuten, daß die damals - wahrscheinlich aus Proben von Urospora wormskioldit - isolierten Fäden zu Hormiscia neglecta gehörten, zwischen denen sie auch im Frühjahr 1966 gefunden wurden. In ihrer Breite entsprechen die zu dieser Jahreszeit gesammelten Fäden von Hormiscia neglecta ganz denen von "Urospora bangioides". Bei beiden Arten verändert sich im Laufe des Jahres die Fadendicke im gleichen Sinne: die im Juli und August im obersten Litoral gesammelten Fäden sind nur etwa $50 \mu$ dick. Wie dieses Beispiel deutlich zeigt, sind morphologische Merkmale für die Trennung der Arten dieses Formenkreises nicht geeignet.

Das Kulturexperiment als Bestimmungsmethode ist sicherlich unbequem, es wird sich aber in vielen Fällen nicht umgehen lassen. Dabei ist es wichtig, aus einer inhomogen erscheinenden Probe einzelne Codiolum-Pflanzen oder Fäden für die Versuche zu isolieren. Im allgemeinen bilden die Fäden bei $15^{\circ} \mathrm{C}$ rasch Zoosporangien. Die Codiolum-Zellen können in Kultur bei $5^{\circ} \mathrm{C}$ zur Reife gebracht werden und entleeren sich meistens von selbst. Es ist aber auch möglich, einzelne fertile Sporophyten zu öffnen, um zu Zoosporen zu gelangen. Die Zoosporen entwickeln sich bei $15^{\circ} \mathrm{C}$ ent- 
weder zu Zwergpflanzen oder zu Fäden. Damit wird ihre Zugehörigkeit zur Gattung Urospora beziehungsweise Hormiscia geklärt. Besonders erstrebenswert wäre es, in den Kulturen Geschlechtspflanzen von Hormiscia zu erzielen, um auch Besonderheiten der Gameten und der Sporophytengeneration für die Kennzeichnung der Arten verwenden zu können.

\section{ZUSAMMENFASSUNG}

1. Hormiscia penicilliformis wächst bei Helgoland häufig in Gemeinschaft mit einer habituell ganz ähnlichen, bisher nicht erkannten Art, die als Hormiscia neglecta beschrieben wird.

2. Hormiscia neglecta ist durch Merkmale ihrer Fadenbasis sowie Größe und Gestalt ihrer Gameten und Sporophyten von Hormiscia penicilliformis unterschieden.

3. Codiolum gregarium, von BRAUN (1855) nach Material von Helgoland beschrieben, ist der Sporophyt von Hormiscia neglecta. Er wurde in allen Stadien seiner Entwicklung am Standort beobachtet und abgebildet.

4. Sporophyten von Hormiscia penicilliformis konnten in der Natur nicht gefunden werden. Dagegen gedeihen die Sporophyten beider Arten gut in Kultur.

5. Die früher als Urospora bangioides bezeichnete Form ist zumindest im Gebiet von Helgoland keine selbständige Art, sondern eine durch die Umweltfaktoren bedingte Modifikation von Hormiscia penicilliformis.

Mit besonderem Dank erkenne ich die unermüdliche und sorgfältige Mitarbeit meines technischen Assistenten, Herrn P.-H. SAHLING, an. Er hat das Gebiet um Helgoland auf zahlreichen Exkursionen durchsucht, Hunderte von Proben gesichtet und eine umfangreiche Sammlung von Photographien und Präparaten geschaffen.

\section{ZITIERTE LITERATUR}

Börgesen, F., 1902. The marine algae of the Faeröes. Aus: Botany of the Faeröes. Det Nordiske Forl., København, Pt 2, 339-532.

Braun, A., 1855. Algarum unicellularium genera nova et minus cognita. Engelmann, Leipzig, $111 \mathrm{pp}$.

Collins, F. S., 1909. The green algae of North America. Tufts Coll. Stud. (Sci. ser.) 2 (3), 79-480.

FrIES, E. M., 1835. Flora scanica (Corpus florarum provincialium Sueciae. 1). Palmblad, Uppsala, 394 pp.

Hanic, L. A., 1965. Life history studies on Urospora and Codiolum from Southern British Columbia. Vancouver, B.C., Thesis of the Univ. of Br. Columbia, $152 \mathrm{pp}$.

JoRdE, I., 1933. Untersuchungen über den Lebenszyklus von Urospora AREscr. und Codiolum A. Braun. Nytt Mag. Naturvid. 73, 1-19.

Kormmann, P., 1961. Uber Codiolum und Urospora. Helgoländer wiss. Meeresunters. 8, 42-57.

- 1964. Zur Biologie von Spongomorpba aeruginosa (Linnaeus) van DEN Hoek. Helgoländer wiss. Meeresunters. 11, 200-208.

PrInTz, H., 1926. Die Algenvegetation des Trondhjemsfjordes. Skr, norske Vidensk-Akad. (Mat.-nat. Kl.), Jg. 1926, No 5, 1-273. 